Pak. j. sci. ind. res. Ser. B: biol. sci. 2020 63B(1) 9-16

\title{
Biochemical Profile of Olive Leaves Grown in "Olive Valley" Pakistan
}

\author{
Fakhara Khanuma*, Tahir Zahoor ${ }^{a}$, Muhammad Issa Khan ${ }^{a}$, Muhammad Asghar ${ }^{b}$, \\ ${ }^{a}$ National Institute of Food Science and Technology, University of Agriculture, Faisalabad, Pakistan \\ ${ }^{b}$ Department of Biochemistry, University of Agriculture, Faisalabad, Pakistan
}

(received February 22, 2018; revised August 21, 2018; accepted August 30, 2018)

\begin{abstract}
This study was designed to determine the best olive variety for cultivation in Pakistan. The leaves of eight locally grown varieties were analyzed for levels of moisture, total solids, ash, fat, fibre, protein, $\mathrm{K}, \mathrm{Ca}, \mathrm{Mg}, \mathrm{Na}, \mathrm{Fe}, \mathrm{Mn}, \mathrm{Zn}$ and $\mathrm{Cu}$ levels. Leaves were then subjected to solvent extraction with each of water ratio in, $75 \%$ ethanol, $75 \%$ methanol, $95 \%$ ethanol and $95 \%$ methanol. The extract yield was significantly highest for $75 \%$ ethanol extract of all varieties. Significantly highest polyphenol and flavonoid contents were examined for Gemlik leaves extracted with $75 \%$ ethanol. We recommend cultivation of Gemlik in Pakistan and extraction of bioactive compounds from olive leaves will be the most efficient with a binary solution of $75 \%$ ethanol in water. Further studies should be planned to assess antioxidant, antimicrobial and food preservation properties of olive leaf extract from locally grown varieties.
\end{abstract}

Keywords: olive, leaf extract, phenol, flavonoid

\section{Introduction}

Olive (Olea europaea), one of the extensively cultivated crops in the World is grown for its fruits, wood and leaves. Olives cultivated in the Mediterranean region account for $98 \%$ of the World's total olive production (Ghanbari et al., 2012). Olive is grown for its fruits which are consumed as table olives and used for making olive oil. Substantial quantity of olive leaves is generated during fruit harvesting and pruning of olive trees. Commonly, the leaves are burned and composted on the farms as waste or used as animal feed ( De Leonardis et al., 2008; Gorinstein et al., 2003). However, the leaves are also used for making olive leaf extract, olive leaf tea (Jilani et al., 2016) and a source of traditional medicines all over the Mediterranean region (Ghanbari et al., 2012).

Deterioration of vital chemicals, lipid oxidation, changes in flavour and colour are the most important challenges in food storage and cosmetic industries (Kiritsakis et al., 2010). To reduce such problems, synthetic additives such as tertiary butyl hydroquinone, butylated hydroxytoluene and butylated hydroxyanisole are used (Pazos et al., 2008). Albeit, these synthetic food additives are stable, inexpensive and effective yet their use is associated with some adverse health phenomenon such as, carcinogenesis and toxicological effects (Cheng et al., 2016; Kiritsakis et al., 2010). Such drawbacks

*Author for correspondence; E-mail: fakharakhan@yahoo.com of synthetic food additives have led to increased use of natural sources as food additives, for the last two decades. Many plant polyphenols and bioactive compounds have antimicrobial, antioxidant, anticarcinogenic, antiviral and health promoting properties (Sanchez-Moreno, 2002). Such plant bioactive compounds are preferred to be used in cosmetics, food storage and pharmaceutical industries as additive, preservatives and dietary supplements.One such candidate is olive leaf extract (OLE) because OLE can improve oxidative stability and antioxidant capacity of edible oils along with health benefits (Salta et al., 2007). Main bioactive compounds in OLE are polyphenols, flavonoids, secoiridoids, triterpenes, hydroxytyrosol (Yateem et al., 2014). To benefit from such compounds of leaves and fruits, the compounds must be extracted. Solvent extraction is a cheap, efficient and commonly used process in food, pharmaceutical and cosmetic industries (Murphy and Sies, 1990). However, extraction efficiency depends on the chemical nature of polyphenols to be extracted, variety, the environmental and geographical impact of varieties, extraction solvent and future applications of extract. Hence there is a need to know the best extraction solvent (ES) for our locally grown olive varieties to facilitate olive production in the country.

Annual import of oil seed in Pakistan is USD 2.2 billion for import of $70 \%$ of total edible consumption. Pakistan has 3.17 million hectares of land with potential of olive 
production (Dhakku, 2012). Converting wild olive to commercial olive varieties in natural habitats and establishment of new olive plantations will have great impact on the economic prosperity of people. Presence of wild olive (Kahu) all around in Pothohar area of Pakistan indicates the possibility of successful cultivation and domestication of olive in this area. The provincial government of Punjab has declared the Pothohar area as "Olive Valley" and incentives for promoting olive production in the area have been launched (Dhakku, 2012).

Keeping in view the huge potential of olive cultivation in Pakistan, there is a need to determine the best variety/varieties which should be cultivated in the country. Also, there is a need to develop suitable costeffective industry-based technology for the extraction of highvalue active compounds from different indigenous olive varieties available in the country. Therefore, the current study was designed with the objectives of (1) recommend some olive variety/varieties for cultivation in Pakistan and (2) determine the most efficient method for extraction of bioactive compound from our local olive varieties.

\section{Materials and Methods}

The study was completed at the University of Agriculture Faisalabad, Pakistan (National Institute of Food Science and Technology and Department of Biochemistry) and Washington State University, Pullman, United States (Department of Food Science and Human Nutrition, and Department of Biological System Engineering).The olive leaves were procured from Barani Agriculture Research Institute, Chakwal, Pakistan. The leaves were from eight locally grown olive varieties: Gemlik, Manzanilla, Sevillano, BARI-Zaitoon (BARI-1), BARIZaitoon (BARI-2), Earlik, Azerbaijan and Hamdi.

Olive leaves were analyzed for their proximate composition and results were taken as means of triplicate. Samples were evaluated for moisture (method no. 4415A), ash (method no. 08-01), protein (method no 4610), fibre content (method no. 32-10) and crude fat(method no. 30-25) following(AACC, 2000). Nitrogen-free extract (NFE) was calculated using the formula: NFE $\%=100-$ (moisture contents $\%+$ crude protein $\%+$ crude fat $\%+$ crude fibre $\%+$ ash $\%$ ). Mineral profile was determined following AOAC method No. 3.014-016(AOAC, 1990). Ca, Fe, Cu, Zn and $\mathrm{Mn}$ were estimated using Atomic Absorption Spectrophotometer (AA240 Varian K, Australia), while $\mathrm{Na}$ and $\mathrm{K}$ were determined by Flame photometer.

Olive leaf extract was obtained following the protocol of Yaghoubi et al. (2007) with some modifications. 10 $\mathrm{g}$ of each olive variety's powder was extracted for two hours with $200 \mathrm{ml}$ of aqueous solutions of $75 \%$ ethanol, 95 ethanol, $75 \%$ methanol, 95 methanol and water at $38^{\circ} \mathrm{C}$ using a thermo-shaker fixed at $180 \mathrm{rpm}$. Then the samples were centrifuged at $5000 \mathrm{rpm}$ for 15 minutes and the supernatant was carried to a rotary evaporatour $\left(38^{\circ} \mathrm{C}, 120 \mathrm{rpm}\right)$ to remove any solvent. The remaining aqueous solutions were lyophilized at $-50^{\circ} \mathrm{C}$ and 0.028 mbar. Percent (w/w) extraction yields of each variety was recorded. This crude extract was refrigerated in glass bottles until further analysis.

Total phenolic contents of OLE were determined using Folin-Ciocalteu method with the modification of Lako et al. (2007). TPC of OLE was calculated with formula $\mathrm{C}(\mathrm{GAEq})=\mathrm{C} \times \mathrm{V} / \mathrm{M}$; where $\mathrm{C}=$ concentration determined by standard curve, $\mathrm{V}=$ volume of sample used, $\mathrm{M}=$ mass of sample used Colourimetric assay was used to measure the total flavonoids of OLE following (Cao et al., 2013), using catechin as standard. Total flavonoids content in the extract was expressed in $\mathrm{mg}$ catechin equivalent per $\mathrm{g}$ of dried olive leaves ( $\mathrm{mg} \mathrm{CE} / \mathrm{g} \mathrm{DOL}$ ).

The data were statistically analyzed through two-way analysis of variance (ANOVA) under completely randomized design (CRD), using university edition of Statistical Analyses System (SAS) software. All analyses were done $\alpha=0.05$.

\section{Results and Discussion}

Proximate analysis of olive leaves. Moisture, total solids, ash, fibre, protein, NFE and $\mathrm{pH}$ varied significantly among varieties, however, fat did not differ significantly among varieties (Table 1). BARI-2 showed significantly highest moisture content (55.96 $2.21 \%)$ whereas, Manzanilla had the lowest moisture contents

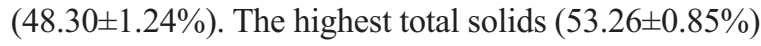
were observed in Manzanilla and the lowest $(46.46 \pm 1.62 \%)$ were found in BARI-1. The mean fat contents were the lowest $(1.23 \pm 0.40 \%)$ in Gemlik and highest $(1.53 \pm 0.11 \%)$ in BARI-2. Percentage ash contents was highest $(1.05 \pm 0.02 \%)$ in Gemlik, while and the lowest $(0.69 \pm 0.02 \%)$ in BARI-1. Highest fibre and protein percentage $(2.56 \pm 0.05 \%$ and $2.73 \pm 0.15 \%)$ 
Table 1. Mean $\pm \mathrm{SE}$ values for proximate composition of olive leaves from the studies varieties

\begin{tabular}{llllllll}
\hline \hline & Moisture $\%$ & Total solid $\%$ & Ash $\%$ & Fat $\%$ & Fibre $\%$ & Protein $\%$ & NFE $\%$ \\
\hline BARI-2 & $55.96 \pm 2.21^{\mathrm{a}}$ & $47.10 \pm 1.41^{\mathrm{a}}$ & $0.86 \pm 0.03 \mathrm{ab}$ & $1.53 \pm 0.11^{\mathrm{a}}$ & $2.06 \pm 0.05^{\mathrm{b}}$ & $2.20 \pm 0.20^{\mathrm{cd}}$ & $37.36 \pm 1.73^{\mathrm{c}}$ \\
BARI-1 & $55.93 \pm 1.23^{\mathrm{a}}$ & $46.46 \pm 1.62^{\mathrm{a}}$ & $0.69 \pm 0.02^{\mathrm{a}}$ & $1.40 \pm 0.26^{\mathrm{a}}$ & $2.23 \pm 0.15^{\mathrm{ab}}$ & $2.03 \pm 0.05^{\mathrm{d}}$ & $37.70 \pm 1.36^{\mathrm{c}}$ \\
Gemlik & $52.86 \pm 0.85^{\mathrm{ab}}$ & $46.83 \pm 1.62^{\mathrm{a}}$ & $1.05 \pm 0.02^{\mathrm{ab}}$ & $1.23 \pm 0.40^{\mathrm{a}}$ & $2.36 \pm 0.20^{\mathrm{ab}}$ & $2.46 \pm 0.05^{\mathrm{abcd}}$ & $40.01 \pm 0.95^{\mathrm{bc}}$ \\
Hamdi & $52.40 \pm 2.12^{\mathrm{abc}}$ & $49.10 \pm 1.56^{\mathrm{ab}}$ & $0.81 \pm 0.05^{\mathrm{b}}$ & $1.36 \pm 0.05^{\mathrm{a}}$ & $2.53 \pm 0.15^{\mathrm{a}}$ & $2.40 \pm 0.26^{\mathrm{abcd}}$ & $40.48 \pm 2.09^{\mathrm{abc}}$ \\
Azerbaijan & $50.46 \pm 1.45^{\mathrm{bc}}$ & $51.63 \pm 0.80^{\mathrm{bc}}$ & $0.86 \pm 0.01^{\mathrm{b}}$ & $1.33 \pm 0.15^{\mathrm{a}}$ & $2.56 \pm 0.11^{\mathrm{a}}$ & $2.26 \pm 0.20^{\mathrm{bcd}}$ & $42.5 \pm 1.47^{\mathrm{ab}}$ \\
Earlik & $50.23 \pm 1.30^{\mathrm{bc}}$ & $51.13 \pm 1.83^{\mathrm{bc}}$ & $0.96 \pm 0.04^{\mathrm{ab}}$ & $1.26 \pm 0.20^{\mathrm{a}}$ & $2.56 \pm 0.05^{\mathrm{a}}$ & $2.73 \pm 0.15^{\mathrm{a}}$ & $42.23 \pm 1.35^{\mathrm{ab}}$ \\
Sevillano & $48.70 \pm 1.15^{\mathrm{bc}}$ & $51.90 \pm 0.70^{\mathrm{bc}}$ & $0.86 \pm 0.02^{\mathrm{ab}}$ & $1.50 \pm 0.20^{\mathrm{a}}$ & $2.43 \pm 0.05^{\mathrm{ab}}$ & $2.50 \pm 0.10^{\mathrm{abc}}$ & $44.00 \pm 1.25^{\mathrm{ab}}$ \\
Manzanilla & $48.36 \pm 1.24^{\mathrm{c}}$ & $53.26 \pm 0.85^{\mathrm{c}}$ & $0.93 \pm 0.03^{\mathrm{ab}}$ & $1.26 \pm 011^{\mathrm{a}}$ & $2.23 \pm 0.15^{\mathrm{ab}}$ & $2.66 \pm 0.15^{\mathrm{ab}}$ & $41.53 \pm 1.49^{\mathrm{a}}$ \\
\hline \hline
\end{tabular}

Different letters as superscript indicate significant differences at alpha $=0.05$.

were observed in Earlik, while the lowest values $(2.06 \pm 0.05 \%$ and $2.03 \pm 0.05 \%)$ in BARI- 2 and BARI1 , accordingly. Moreover, BARI-2 showed the lowest $(37.36 \pm 1.73 \%)$ and Sevillano showed the highest $(44.00 \pm 1.25 \%)$ nitrogen-free extract percentage.

Mineral contents. The levels of all minerals varied significantly among the verities (Table 2). Gemlik showed the highest calcium contents $(20.36 \pm 0.32 \mathrm{~g} / \mathrm{kg})$ and BARI-2 showed the lowest Calcium contents $12.90 \pm 0.20 \mathrm{~g} / \mathrm{kg}$. Similarly, Gemlik was recorded with highest contents of magnesium and zinc $(2.73 \pm 0.20$ $\mathrm{g} / \mathrm{kg}$ and $28.23 \pm 0.41 \mathrm{mg} / \mathrm{kg}$ ). The highest sodium contents $(426.7 \pm 20.8)$ was found in Sevillano, whereas the lowest was found in BARI-1. The variety Manzanilla showed the significantly highest concentration of magnesium, iron, and copper $(1.93 \pm 0.25 \mathrm{~g} / \mathrm{kg}$, $73.56 \pm 1.51$, and $25.60 \pm 0.36 \mathrm{mg} / \mathrm{kg}$, respectively). The lowest calcium, magnesium and iron concentration was found in BARI-2 $(12.90 \pm 0.20 \mathrm{~g} / \mathrm{kg}, 0.80 \pm 0.11 \mathrm{~g} / \mathrm{kg}$ and $54.63 \pm 0.70 \mathrm{mg} / \mathrm{kg}$, respectively).

Table 2. Mean \pm SE values for the mineral contents for olive leaves of the studies varieties

\begin{tabular}{lllllllll}
\hline \hline Varieties & $\mathrm{Kg} / \mathrm{kg}$ & $\mathrm{Ca} \mathrm{g} / \mathrm{kg}$ & $\mathrm{Mg} \mathrm{g} / \mathrm{kg}$ & $\mathrm{Na} \mathrm{mg} / \mathrm{kg}$ & $\mathrm{Fe} \mathrm{mg} / \mathrm{kg}$ & $\mathrm{Mn} \mathrm{mg} / \mathrm{kg}$ & $\mathrm{Zn} \mathrm{mg} / \mathrm{kg}$ & $\mathrm{Cu} \mathrm{mg} / \mathrm{kg}$ \\
\hline BARI-2 & $7.73 \pm 0.37^{\mathrm{cd}}$ & $12.90 \pm 0.20^{\mathrm{e}}$ & $0.80 \pm 0.11^{\mathrm{e}}$ & $306.7 \pm 5.7^{\mathrm{c}}$ & $54.63 \pm 0.70^{\mathrm{e}}$ & $26.49 \pm 1.22^{\mathrm{c}}$ & $22.66 \pm 3.40^{\mathrm{bc}}$ & $14.50 \pm 0.20^{\mathrm{de}}$ \\
BARI-1 & $7.70 \pm 0.43^{\mathrm{d}}$ & $13.70 \pm 0.20^{\mathrm{e}}$ & $1.26 \pm 0.40^{\mathrm{de}}$ & $286.7 \pm 15.3^{\mathrm{c}}$ & $57.83 \pm 1.01^{\mathrm{de}}$ & $27.95 \pm 0.48^{\mathrm{bc}}$ & $18.33 \pm 0.47^{\mathrm{d}}$ & $10.93 \pm 0.25^{\mathrm{f}}$ \\
Gemlik & $9.93 \pm 0.47^{\mathrm{a}}$ & $20.36 \pm 0.32^{\mathrm{a}}$ & $2.73 \pm 0.20^{\mathrm{a}}$ & $410.0 \pm 45.9^{\mathrm{ab}}$ & $72.0 \pm 4.68^{\mathrm{ab}}$ & $32.86 \pm 1.62^{\mathrm{a}}$ & $28.23 \pm 0.41^{\mathrm{a}}$ & $13.40 \pm 0.30^{\mathrm{e}}$ \\
Hamdi & $8.86 \pm 0.61^{\mathrm{abcd}}$ & $16.00 \pm 0.55^{\mathrm{cd}}$ & $2.00 \pm 0.10 \mathrm{~b}^{\mathrm{c}}$ & $416.7 \pm 15.3^{\mathrm{a}}$ & $66.53 \pm 3.15^{\mathrm{bc}}$ & $28.38 \pm 0.70^{\mathrm{bc}}$ & $24.50 \pm 0.20^{\mathrm{b}}$ & $21.10 \pm 0.79^{\mathrm{b}}$ \\
Azerbaijan & $8.40 \pm 0.20^{\mathrm{bcd}}$ & $15.43 \pm 0.25^{\mathrm{d}}$ & $1.40 \pm 0.17^{\mathrm{cde}}$ & $296.7 \pm 5.7^{\mathrm{c}}$ & $63.26 \pm 2.30^{\mathrm{cd}}$ & $26.46 \pm 0.76^{\mathrm{c}}$ & $19.66 \pm 0.45^{\mathrm{cd}}$ & $11.70 \pm 0.20^{\mathrm{f}}$ \\
Earlik & $8.93 \pm 0.47^{\mathrm{abc}}$ & $16.56 \pm 0.30^{\mathrm{c}}$ & $1.46 \pm 0.15^{\mathrm{cd}}$ & $373.3 \pm 30.5^{\mathrm{ab}}$ & $63.80 \pm 1.25^{\mathrm{cd}}$ & $27.87 \pm 1.51^{\mathrm{bc}}$ & $23.80 \pm 0.65^{\mathrm{b}}$ & $15.13 \pm 0.40^{\mathrm{d}}$ \\
Sevillano & $8.86 \pm 0.20^{\mathrm{abcd}}$ & $17.60 \pm 0.36^{\mathrm{b}}$ & $2.35 \pm 0.18^{\mathrm{ab}}$ & $426.7 \pm 20.8^{\mathrm{a}}$ & $58.90 \pm 1.37^{\mathrm{de}}$ & $29.63 \pm 0.18^{\mathrm{b}}$ & $21.71 \pm 0.47^{\mathrm{bcd}}$ & $16.90 \pm 0.40^{\mathrm{c}}$ \\
Manzanilla & $9.50 \pm 0.52^{\mathrm{b}}$ & $18.50 \pm 0.20^{\mathrm{b}}$ & $1.93 \pm 0.25^{\mathrm{bc}}$ & $350.0 \pm 10.0^{\mathrm{bc}}$ & $73.56 \pm 1.51^{\mathrm{a}}$ & $34.60 \pm 0.75^{\mathrm{a}}$ & $21.60 \pm 0.81^{\mathrm{bcd}}$ & $25.60 \pm 0.36^{\mathrm{a}}$ \\
\hline \hline
\end{tabular}

Different letters as superscript indicate significant differences at alpha $=0.05$

Table 3. Mean \pm SE values for the percent crude extract yield from leaves of the studies olive varieties

\begin{tabular}{|c|c|c|c|c|c|c|c|c|c|}
\hline \multicolumn{4}{|c|}{ Extraction solvents } & \multicolumn{6}{|c|}{ Olive leaf varieties } \\
\hline & Gemlik & Earlik & Manzanilla & Sevillano & Hamdi & Azerbaijan & BARI-2 & BARI-1 & Mean \\
\hline $\begin{array}{l}\text { Ethanol } \\
(75 \%)\end{array}$ & $18.13^{\mathrm{a}}$ & $15.4^{\mathrm{c}}$ & $13.9^{\mathrm{e}}$ & $12.6^{\mathrm{f}}$ & $12^{\text {fghi }}$ & $12.2^{\mathrm{fgi}}$ & $10.3^{\mathrm{klm}}$ & $9.8^{\mathrm{m}}$ & $13^{\mathrm{A}}$ \\
\hline $\begin{array}{l}\text { Methanol } \\
(75 \%)\end{array}$ & $16.6^{\mathrm{b}}$ & $14.1^{\mathrm{de}}$ & $11.3^{\mathrm{ij}}$ & $11.7^{\mathrm{hi}}$ & $10.8^{\mathrm{jk}}$ & $11.2 \mathrm{ij}$ & $9.06^{\mathrm{n}}$ & $9.03^{\mathrm{n}}$ & $11.7^{\mathrm{B}}$ \\
\hline $\begin{array}{l}\text { Ethanol } \\
(95 \%)\end{array}$ & $14.8^{\mathrm{cd}}$ & $12.5^{\mathrm{fg}}$ & $10.6 \mathrm{jkl}$ & $10.5^{\mathrm{jklm}}$ & $10.1^{\mathrm{klm}}$ & $10.8^{\mathrm{jk}}$ & $8.7^{\mathrm{n}}$ & $8.4^{\text {no }}$ & $10.8^{\mathrm{C}}$ \\
\hline $\begin{array}{l}\text { Methanol } \\
(95 \%)\end{array}$ & $14.0^{\mathrm{e}}$ & $11.8^{\mathrm{ghi}}$ & 11.6hi & $10.0^{\mathrm{lm}}$ & $10.1^{\mathrm{klm}}$ & $9.96^{\mathrm{lm}}$ & $8.4^{\text {no }}$ & $7.7^{\circ}$ & $10.5^{\mathrm{D}}$ \\
\hline Mean & $15.9^{\mathrm{A}}$ & $13.4^{\mathrm{B}}$ & $11.8^{\mathrm{C}}$ & $11.2^{\mathrm{D}}$ & $10.8^{\mathrm{E}}$ & $11^{\mathrm{DE}}$ & $9.1^{\mathrm{F}}$ & $8.7^{\mathrm{G}}$ & 11.5 \\
\hline
\end{tabular}

Different small and capital letters as superscript indicate respective significant differences at alpha $=0.05$ 
Crude extract yield. The overall mean values for extract varied from 8.7 to $15.9 \%$ (Table 3 ). The significantly highest yield of soluble components was observed in Gemlik (15.9\%) followed by the yield for Earlik, Manzanilla, Sevillano, Hamdi, Azerbaijan, and BARI-2 $(13.4,11.8,11.2,10.8,11$ and $9.1 \%$, respectively). BARI-1 showed lowest (8.7\%) extract yield. Among extraction solvents: $75 \%$ ethanol exhibited significantly highest yield (13\%), whereas, $75 \%$ methanol and $95 \%$ ethanol showed extraction yield of 11.7 and $10.8 \%$. However, the minimum extract yield of $10.5 \%$ was detected with $95 \%$ methanol. Water extract was not dried to measured yield for any variety.
Interaction of varieties and extraction treatments also showed highly significant variation for yield of OLE (Table 3). The values for extract yield fell within the range: $7.7-8.13 \%$. Significantly highest yield $(18.13 \%)$ was observed for Gemlik leaves extracted with $75 \%$ ethanol while BARI-1 extracted with $95 \%$ methanol presented the lowest $(7.7 \%)$ yield.

Total phenolic contents. Total phenolic contents (TPC) varied from $38.47 \pm 2.28$ to $126.59 \pm 9.42 \mathrm{mg} \mathrm{GAEq} / \mathrm{g}$ of extract (Table 4). Significantly highest (126.59 \pm 9.42 $\mathrm{mg} \mathrm{GAEq} / \mathrm{g}$ ) phenolic contents were found in Gemlik followed by Manzanilla, Sevillano, Hamdi, Earlik,

Table 4. Mean \pm SE values for TPC (mg GAEq/g extract) from leaves of the studied olive varieties. Different small and capital letters as superscript indicate respective significant differences at alpha $=0.05$

\begin{tabular}{llllllllll}
\hline \hline & Gemlik & Manzanilla & Sevillano & Earlik & Azerbaijan & Hamdi & BARI-1 & BARI-2 & Means \\
\hline Ethanol & 156.01 & 123.79 & 90.94 & 73.44 & 74.71 & 90.94 & 48.90 & 43.78 & 87.81 \\
$(75 \%)$ & $\pm 1.70^{\mathrm{a}}$ & $\pm 1.09^{\mathrm{e}}$ & $\pm 0.85^{\mathrm{h}}$ & $\pm 1.64^{\mathrm{j}}$ & $\pm 3.69^{\mathrm{j}}$ & $\pm 0.85^{\mathrm{h}}$ & $\pm 0.24^{\mathrm{o}}$ & $\pm 0.19^{\mathrm{pq}}$ & $\pm 7.31^{\mathrm{A}}$ \\
Methanol & 149.54 & 116.19 & 86.00 & 65.90 & 66.21 & 86.00 & 47.11 & 41.84 & 82.35 \\
$(75 \%)$ & $\pm 0.76^{\mathrm{b}}$ & $\pm 1.92^{\mathrm{f}}$ & $\pm 0.80^{\mathrm{i}}$ & $\pm 0.35^{\mathrm{k}}$ & $\pm 1.80^{\mathrm{k}}$ & $\pm 0.80^{\mathrm{i}}$ & $\pm 0.39^{\text {op }}$ & $\pm 0.56^{\mathrm{q}}$ & $\pm 7.04^{\mathrm{B}}$ \\
Ethanol & 139.89 & 108.42 & 81.77 & 60.67 & 61.18 & 81.77 & 37.11 & 43.78 & 76.82 \\
$(95 \%)$ & $\pm 3.05^{\mathrm{c}}$ & $\pm 0.37^{\mathrm{g}}$ & $\pm 0.97^{\mathrm{i}}$ & $\pm 0.561^{\mathrm{m}}$ & \pm 1.311 & $\pm 0.97^{\mathrm{i}}$ & $\pm 0.39^{\mathrm{r}}$ & $\pm 0.19^{\mathrm{pq}}$ & $\pm 6.68^{\mathrm{C}}$ \\
Methanol & 128.86 & 105.05 & 75.18 & 56.92 & 58.06 & 75.18 & 32.43 & 35.07 & 70.84 \\
$(95 \%)$ & $\pm 1.84^{\mathrm{d}}$ & $\pm 2.15^{\mathrm{g}}$ & $\pm 2.55^{\mathrm{j}}$ & $\pm 1.45^{\mathrm{mn}}$ & $\pm 2.391^{\mathrm{m}}$ & $\pm 2.55^{\mathrm{j}}$ & $\pm 0.33^{\mathrm{S}}$ & $\pm 0.68^{\mathrm{rs}}$ & $\pm 6.48^{\mathrm{D}}$ \\
Water & 58.66 & 53.62 & 47.69 & 46.46 & 46.40 & 45.02 & 26.80 & 30.91 & 44.44 \\
& $\pm 1.841^{\mathrm{m}}$ & $\pm 1.76^{\mathrm{n}}$ & $\pm 1.36^{\text {op }}$ & $\pm 1.30^{\text {op }}$ & $\pm 0.59^{\mathrm{op}}$ & $\pm 2.69^{\mathrm{opq}}$ & $\pm 1.57^{\mathrm{t}}$ & $\pm 0.29^{\mathrm{st}}$ & $\pm 2.12^{\mathrm{E}}$ \\
Means & 126.59 & 101.42 & 76.32 & 60.68 & 61.31 & 75.78 & 38.47 & 39.07 & 72.45 \\
& $\pm 9.42^{\mathrm{A}}$ & $\pm 6.64^{\mathrm{B}}$ & $\pm 4.10^{\mathrm{C}}$ & $\pm 2.45^{\mathrm{D}}$ & $\pm 2.63^{\mathrm{D}}$ & $\pm 4.38^{\mathrm{C}}$ & $\pm 2.28^{\mathrm{E}}$ & $\pm 1.39^{\mathrm{E}}$ & \pm 3.07 \\
\hline \hline
\end{tabular}

Table 5. Mean \pm SE values for flavonoid contents ( $\mathrm{mg} \mathrm{CE} / \mathrm{g} \mathrm{DOL}$ ) from leaves of the studied olive varieties. Different small and capital letters as superscript indicate respective significant differences at alpha $=0.05$

\begin{tabular}{|c|c|c|c|c|c|c|c|c|c|}
\hline \multirow{2}{*}{$\begin{array}{l}\text { Method of } \\
\text { extraction }\end{array}$} & \multicolumn{9}{|c|}{ Olive varieties } \\
\hline & Gemlik & Manzanilla & Sevillano & Earlik & Azrbaijan & Hamdi & BARI-1 & BARI-2 & Mean \\
\hline $\begin{array}{l}\text { Ethanol } \\
(75 \%)\end{array}$ & $\begin{array}{l}147.68 \\
\pm 4.14^{\mathrm{a}}\end{array}$ & $\begin{array}{l}118.12 \\
\pm 2.00^{\mathrm{c}}\end{array}$ & $\begin{array}{l}85.84 \\
\pm 1.44^{\text {hi }}\end{array}$ & $\begin{array}{l}107.31 \\
\pm 1.91^{\mathrm{de}}\end{array}$ & $\begin{array}{l}76.36 \\
\pm 1.15^{\mathrm{jk}}\end{array}$ & $\begin{array}{l}94.80 \\
\pm 2.69^{\mathrm{f}}\end{array}$ & $\begin{array}{l}60.82 \\
\pm 1.03^{\text {nopq }}\end{array}$ & $\begin{array}{l}63.70 \\
\pm 0.91^{\mathrm{mn}}\end{array}$ & $\begin{array}{l}94.32 \\
\pm 5.76^{\mathrm{A}}\end{array}$ \\
\hline $\begin{array}{l}\text { Methanol } \\
(75 \%)\end{array}$ & $\begin{array}{l}139.28 \\
\pm 1.93^{\mathrm{b}}\end{array}$ & $\begin{array}{l}110.47 \\
\pm 2.93^{\mathrm{d}}\end{array}$ & $\begin{array}{l}82.44 \\
\pm 1.33^{\mathrm{i}}\end{array}$ & $\begin{array}{l}102.41 \\
\pm 1.46 \mathrm{e}\end{array}$ & $\begin{array}{l}68.15 \\
\pm 1.641^{\mathrm{m}}\end{array}$ & $\begin{array}{l}88.53 \\
\pm 0.61^{\text {gh }}\end{array}$ & $\begin{array}{l}57.83 \\
\pm 1.63^{\text {opqr }}\end{array}$ & $\begin{array}{l}55.66 \\
\pm 0.90^{\text {qrs }}\end{array}$ & $\begin{array}{l}88.09 \\
\pm 5.59^{\mathrm{B}}\end{array}$ \\
\hline $\begin{array}{l}\text { Ethanol } \\
(95 \%)\end{array}$ & $\begin{array}{l}112.08 \\
\pm 4.01^{d}\end{array}$ & $\begin{array}{l}96.46 \\
\pm 1.41^{\mathrm{f}}\end{array}$ & $\begin{array}{l}74.31 \\
\pm 2.33^{\mathrm{k}}\end{array}$ & $\begin{array}{l}92.92 \\
\pm 1.54 \mathrm{fg}\end{array}$ & $\begin{array}{l}61.19 \\
\pm 0.81^{\text {nopq }}\end{array}$ & $\begin{array}{l}81.80 \\
\pm 2.13^{\text {aij }}\end{array}$ & $\begin{array}{l}57.15 \\
\pm 1.20^{\mathrm{pqrs}}\end{array}$ & $\begin{array}{l}47.34 \\
\pm 3.21^{\text {tuv }}\end{array}$ & $\begin{array}{l}77.90 \\
\pm 4.35^{\mathrm{C}}\end{array}$ \\
\hline $\begin{array}{l}\text { Methanol } \\
(95 \%)\end{array}$ & $\begin{array}{l}107.97 \\
\pm 2.37^{\mathrm{de}}\end{array}$ & $\begin{array}{l}91.69 \\
\pm 0.66^{\mathrm{fg}}\end{array}$ & $\begin{array}{l}72.79 \\
\pm 3.20^{\mathrm{k} 1}\end{array}$ & $\begin{array}{l}88.92 \\
\pm 0.89 \mathrm{gh}\end{array}$ & $\begin{array}{l}62.90 \\
\pm 2.70^{\mathrm{mno}}\end{array}$ & $\begin{array}{l}73.87 \\
\pm 2.96^{\mathrm{k}}\end{array}$ & $\begin{array}{l}51.92 \\
\pm 1.41^{\text {stu }}\end{array}$ & $\begin{array}{l}45.71 \\
\pm 2.71^{\mathrm{v}}\end{array}$ & $\begin{array}{l}74.47 \\
\pm 4.16^{\mathrm{D}}\end{array}$ \\
\hline Water & $\begin{array}{l}61.89 \\
\pm 0.65^{\text {nop }}\end{array}$ & $\begin{array}{l}52.59 \\
\pm 0.68^{\text {arst }}\end{array}$ & $\begin{array}{l}41.83 \\
\pm 2.38^{V}\end{array}$ & $\begin{array}{l}46.44 \\
\pm 1.63^{\mathrm{uv}} \\
\end{array}$ & $\begin{array}{l}42.65 \\
\pm 0.73^{\mathrm{V}}\end{array}$ & $\begin{array}{l}46.12 \\
\pm 1.23^{\mathrm{V}}\end{array}$ & $\begin{array}{l}33.75 \\
\pm 0.62^{\mathrm{W}}\end{array}$ & $\begin{array}{l}34.76 \\
\pm 0.39^{\mathrm{w}}\end{array}$ & $\begin{array}{l}45.00 \\
\pm 1.83^{\mathrm{E}} \\
\end{array}$ \\
\hline Means & $\begin{array}{l}113.78 \\
\pm 8.11^{\mathrm{A}}\end{array}$ & $\begin{array}{l}93.86 \\
\pm 6.10^{\mathrm{B}}\end{array}$ & $\begin{array}{l}87.60 \\
\pm 5.80^{\mathrm{C}}\end{array}$ & $\begin{array}{l}77.02 \\
\pm 4.60^{\mathrm{D}}\end{array}$ & $\begin{array}{l}71.44 \\
\pm 4.25^{\mathrm{E}}\end{array}$ & $\begin{array}{l}62.25 \\
\pm 3.03^{\mathrm{F}}\end{array}$ & $\begin{array}{l}52.29 \\
\pm 2.63^{\mathrm{G}}\end{array}$ & $\begin{array}{l}49.43 \\
\pm 2.71^{\mathrm{H}}\end{array}$ & 75.96 \\
\hline
\end{tabular}


Azerbaijan and BARI-2. BARI-1 exhibited significantly lowest (38.47 mg GAEq/g) TPC. The extraction solvents for phenolic contents also exhibited significant variation in TPC of OLE, ranging from 44. $44 \pm 2.12$ to $87.81 \pm$ $7.31 \mathrm{mg} \mathrm{GAEq} / \mathrm{g}$. The extraction with $75 \%$ ethanol showed significantly highest (87.81 $\pm 7.31 \mathrm{mg} \mathrm{GAEq} / \mathrm{g})$ TPC followed by $75 \%$ methanol, $95 \%$ ethanol and $95 \%$ methanol. Whereas, water extraction showed significantly lowest $(44.44 \pm 2.12 \mathrm{mg}$ GAEq/g) TPC.The phenolic contents varied significantly from $26.80 \pm 1.57$ (BARI-1 with water) to $156.01 \pm 1.70$ (for Gemlik with $75 \%$ ethanol) $\mathrm{mg} \mathrm{GAEq} / \mathrm{g}$ for cell means of varieties and solvents.

Flavonoid contents. Mean flavonoid contents $(\mathrm{FC})$ of OLE from the varieties varied from $49.43 \pm 2.71$ to $113.78 \pm 8.11 \mathrm{mg} \mathrm{CE} / \mathrm{g}$ extract (Table 5). The highest FC (113.78 $\pm 8.11 \mathrm{mg} \mathrm{CE} / \mathrm{g})$ were observed for Gemlik. Whereas, BARI-2 showed significantly lowest (49.43 $\pm 2.71 \mathrm{mg} \mathrm{CE} / \mathrm{g}$ extract)FC. Among extraction solvents: significantly highest $\mathrm{FC}(94.32 \pm 5.76 \mathrm{mg} \mathrm{CE} / \mathrm{g})$ were observed in $75 \%$ ethanol extract followed by $75 \%$ methanol, $95 \%$ ethanol and $95 \%$ methanol extract. Whereas, significantly lowest FC $(45.00 \pm 1.83 \mathrm{mg} \mathrm{CE} / \mathrm{g})$ were detected for aqueous extract.The cell means values for FC diverged significantly from $33.75 \pm 0.62$ (for BARI-1 with aqueous extract) to $147.68 \pm 4.14$ (for Gemlik with $75 \%$ ethanol) $\mathrm{mg} \mathrm{CE} / \mathrm{g}$ extract, for different combinations of varieties and methods of extraction.

The results of the current study regarding proximate composition of olive leaves are in close conformity of Cavalheiro et al. (2014) who studied the proximate composition of various olive varieties and found the moisture percentage in the range of $46.24 \pm 0.54$ $58.68 \pm 0.07 \%$. While, ash, crude protein, carbohydrates and crude fat contents were in the range of $(2.86 \pm 0.10$ $4.38 \pm 0.18),(5.04 \pm 0.15-12.24 \pm 0.23),(19.56 \pm 0.38$ $42.58 \pm 0.43)$ and (1.05 $\pm 0.11-8.14 \pm 0.24)$ respectively. These values for moisture, total solids, fat and NFE toe the line with our findings of proximate analysis. However, the mean values for ash and protein in our finding are lower than Cavalheiro et al. (2014). It could be due to different ecological factors and variation in genetic makeup of varieties, native varieties inour project versus the varieties in Cavalheiro et al. (2014). The range of proximate analysis values is narrower is our study than that of Cavalheiro et al. (2014). This could be explained because we obtained leaves from trees grown in same area under similar climatic and soil conditions. As our olive trees were grown in same locality, significant variation among mean values of varieties for moisture, total solids, ash, fibre, protein and NFE agree to Al-Ruqaie et al. (2016) who found significant differences in proximate composition of eight exotic olive varieties grown in Saudi arabia. These significant differences among varieties grown under identical condition could be attributed to genetics.

Results of current study regarding mineral contents corroborate with values presented in the literature. Among all minerals studied in this project, levels of $\mathrm{Ca}$ were the highest for all varieties. Roman et al. (2014) also observed that olive leaves exhibited significantly higher calcium contents than other elements. The range in mean values of minerals among varieties is in line with Paskovic et al. (2013) who detected iron, zinc, manganese and copper concentration in the range of 38.00-52.70, 17.80- 30.17, 23.80-33.40 and 5.80$7.17 \mathrm{mg} / \mathrm{kg}$, respectively. The slight differences between our study and previous literature are probably due to the age of plant, environmental conditions, temperature, humidity and soil composition which affect storage of mineral contents in leaves. Our findings of significant differences for mean mineral content among varieties agrees to Toplu et al. (2009) who claimed that cultivars grown under same climatic conditions may differ in minerals composition. Genetics is a probable cause for significant differences in mineral composition among varieties grown under similar condition as in our study.

In our endeavour, significant variation arose in crude extract yield from olive leaves of selected varieties. The significant differences were found among ES and among varieties. Though the interaction effect was significant, however, it could be due to larger data set and has no practical meaning as extract yield with $75 \%$ ethanol was significantly higher than other the yield by other ES, across all varieties. As extraction efficiency can be affected by temperature, solvent/solid ratio, particle size of the solid material, number of extraction steps and extraction time (Chirinos et al., 2007; Lafka et al., 2007), these factors were constant for combination and Vs and ES in our study. Hence the significant difference in mean extract yield among ES are due to solventtype and concentration (Chirinos et al., 2007; Le Floch et al., 1998). Albeitext raction with water may be considered cheaper, yet it is not the most efficient method of extraction as evident form our results. Abaza et al. (2011) concluded that both extraction efficiency and antioxidant capacity of extracted phytochemicals are intensively influenced by the solvent type, polarity 
of solvent and extracted phenol type. Phenolic compounds have polarity. Therefore, highly polar organic solvent is more effective for extraction than non-polar ones. Thus, water being non-polar is not as efficient for extraction as binary compound of water and organic solvents. It may be logical to think that pure organic solvents will be more efficient for extraction of biochemical compounds, it is not the case. Pure organic compounds are toxic as organic compound will deposit on the extract (Oreopoulou, 2003).

Moreover, in our study $95 \%$ solutions of ethanol and methanol were less efficient than $75 \%$ solutions of ethanol and methanol, it has been confirmed by previous studies (Altiok et al., 2008; Oreopoulou, 2003). Water plays an important role in extraction as it facilitates the diffusion of extractable bioactive compounds through the plant cells. Hence, combination of water and solvent extraction promotes extraction due to binary properties (Altiok et al., 2008).

In present study highest TPC was obtained using 75\% ethanol as the extraction solvent. Similarly, Altiok et al. (2008) recovered the highest value of TPC from $70 \%$ aqueous ethanol as compared to other solvents used. In the current study, the mean TPC values ranged from $26.80 \pm 1.57$ to $156.01 \pm 1.70 \mathrm{mg} \mathrm{GAEq} / \mathrm{g}$ DOL, while mean flavonoid values ranged from $33.75 \pm 0.62$ to $147.68 \pm 4.14 \mathrm{mg} \mathrm{CE} / \mathrm{g}$. Contrarily, Cao et al. (2013) determined the flavonoid and phenolic contents of olive varieties native to the Mediterranean region but grown in Sichuan province of China. The mean TPC values ranged from $34.31 \pm 1.22$ to $56.47 \pm 1.19 \mathrm{mg} \mathrm{GAE} / \mathrm{g}$ dry olive leaves for different olive varieties, while the lower range is in proximity to our study, upper ranged shows considerable differences. These differences could be due to differences in varies, topographicallocation and environment of the areas. Another probable reason may be the use of Mediterranean olive varieties in China, hence the plants were grown in non-habitat environment which may have impacted their chemical composition. However, significantly higher mean TPC and FC in our study is toeing the line with another study from Pakistan. Khaliq et al. (2015) determined TPC and FC contents from extract of eight local olive cultivars of Lorallia, Quetta and Zhoab research station of Pakistan. According to their finding the TPC value ranged from $109.6 \pm 3.68-161 \pm 1.9 \mathrm{mg} \mathrm{GAEq} / \mathrm{g}$ of extract while FC varied from $42 \pm 1.97$ to $57 \pm 1.24 \mathrm{mg} / \mathrm{g}$. Like our findings, they also found that Gemlik possessed the highest amount of TPC and FC. Moreover, Salah et al. (2012) found flavonoid concentration in the range of $56.75 \pm 6.72$ to $125.64 \pm 3.36$ which are very close to current studies. Variation in mean TPC and flavonoid values in the current study and previous literature might be due to differences in geographical, varietal, genetic and environmental factors.

\section{Conclusion}

Based on our findings, we recommend that cultivation of Gemlik be favoured over other olive varieties grown in the country especially in Potohar "olive" valley. Moreover, for manufacturing OLE at industrial levels, $75 \%$ solution of ethanol in water should be used for extraction of bioactive compounds. In the present study, all sampling was from the trees grown in one area, further studies are needed to study olive varieties grown under different areas of the country with different growing conditions. Furthermore, we found considerable levels of polyphenols and flavonoid in the varieties grown in Pakistan, studies are needed to determine antioxidant and antimicrobial potential of olive fruits and leaves from locally gown olive varieties.

Conflict of Interest. The authors declare no conflict of interest

\section{References}

AACC, 2000. Approved Methods of the American Association of Cereal Chemists. American Association of Cereal Chemists Approved Methods.

Abaza, L., Youssef, N.B., Manai, H., Haddada, F.M., Methenni, K., Zarrouk, M. 2011. Chétoui olive leaf extracts: influence of the solvent type on phenolics and antioxidant activities. Grasas Y Aceites, 62: 96-104.

Al-Ruqaie, I., Al-Khalifah, N.S., Shanavaskhan, A.E. 2016. Morphological cladistic analysis of eight popular olive (Olea europaea L.) cultivars grown in Saudi arabia using numerical taxonomic system for personal computer to detect phyletic relationship and their proximate fruit composition. Saudi Journal of Biological Sciences, 23: 115-121.

Altiok, E., Bayçin, D., Bayraktar, O., Ülkü, S. 2008. Isolation of polyphenols from the extracts of olive leaves (Olea europaea L.) by adsorption on silk fibroin. Separation and Purification Technology, 62: 342-348.

AOAC, 1990. Approved Methods of the American Association of Cereal Chemists, Association of 
Official Analytical Chemists. Volume 1-2, Technology of Enginering , 1200 pages.

Cao, Z., Meng, F., Zhao, G. 2013. Optimization of extraction process of olive leaves by RSM and evaluation of antioxidant capacity measurements to the mixtures. Journal of Food Science and Engineering, 3, 525.

Cavalheiro, C.V., Rosso, V.D., Paulus, E., Cichoski, A.J., Wagner, R., Menezes, C.R. de, Barin, J.S. 2014. Chemical composition of olive leaves (Olea europaea L.) from the region of Caçapava do Sul, RS, Brazil. Ciência Rural, 44: 1874-1879.

Cheng, J.-S., Chou, C.-T., Liu, Y.-Y., Sun, W.-C., Shieh, P., Kuo, D.-H., Kuo, C.-C., Jan, C.-R., Liang, W.Z. 2016. The effect of oleuropein from olive leaf (Olea europaea) extract on $\mathrm{Ca}^{2+}$ homeostasis, cytotoxicity, cell cycle distribution and ROS signaling in $\mathrm{HepG}^{2}$ human hepatoma cells. Food and Chemical Toxicology, 91: 151-166.

Chirinos, R., Rogez, H., Campos, D., Pedreschi, R., Larondelle, Y. 2007. Optimization of extraction conditions of antioxidant phenolic compounds from mashua (Tropaeolum tuberosum Ruíz \& Pavón) tubers. Separation and Purification Technology, 55: 217-225.

De Leonardis, A., Aretini, A., Alfano, G., Macciola, V., Ranalli, G. 2008. Isolation of a hydroxytyrosolrich extract from olive leaves (Olea Europaea L.) and evaluation of its antioxidant properties and bioactivity. European Food Research and Technology, 226: 653-659.

Dhakku, N.A. 2012. Turning Potohar into olive valley. DAWN.COM. URL http://www.dawn.com/news/ 754766 (accessed 2.7.18).

Ghanbari, R., Anwar, F., Alkharfy, K.M., Gilani, A.-H., Saari, N. 2012. Valuable nutrients and functional bioactives in different parts of olive (Olea europaea L.)-a review. International Journal of Molecular Sciences, 13: 3291-3340.

Gorinstein, S., Martin-Belloso, O., Katrich, E., Lojek, A., Cíž, M., Gligelmo-Miguel, N., Haruenkit, R., Park, Y.-S., Jung, S.-T., Trakhtenberg, S. 2003. Comparison of the contents of the main biochemical compounds and the antioxidant activity of some Spanish olive oils as determined by four different radical scavenging tests. The Journal of Nutritional Biochemistry, 14: 154-159.

Jilani, H., Cilla, A., Barberá, R., Hamdi, M. 2016. Improved bioaccessibility and antioxidant capacity of olive leaf (Olea europaea L.) polyphenols through biosorption on Saccharomyces cerevisiae. Industrial Crops and Products, 84: 131-138.

Khaliq, A., Sabir, S.M., Ahmed, S.D., Boligon, A.A., Athayde, M.L., Jabbar, A.J., Qamar, I., Khan, A. 2015. Antioxidant activities and phenolic composition of Olive (Olea europaea) leaves. Journal of Applied Botany and Food Quality, 88: 16-21.

Kiritsakis, K., Kontominas, M.G., Kontogiorgis, C., Hadjipavlou-Litina, D., Moustakas, A., Kiritsakis, A. 2010. Composition and antioxidant activity of olive leaf extracts from Greek olive cultivars. Journal of the American Oil Chemists 'Society, 87: 369-376.

Lafka, T.-I., Sinanoglou, V., Lazos, E.S. 2007. On the extraction and antioxidant activity of phenolic compounds from winery wastes. Food Chemistry 104: 1206-1214.

Lako, J., Trenerry, V.C., Wahlqvist, M., Wattanapenpaiboon, N., Sotheeswaran, S., Premier, R. 2007. Phytochemical flavonols, carotenoids and the antioxidant properties of a wide selection of Fijian fruit, vegetables and other readily available foods. Food Chemistry, 101: 1727-1741.

Le Floch, F., Tena, M.T., Rios, A., Valcarcel, M. 1998. Supercritical fluid extraction of phenol compounds from olive leaves. Talanta, 46: 1123-1130.

Murphy, M.E., Sies, H. 1990. Visible-range low-level Chemiluminescence in Biological Systems, In: Methods in Enzymology, Elsevier, pp. 595-610.

Oreopoulou, V. 2003. Extraction of Natural Antioxidants. Food Science and Technology-new York-Marcel Dekker- 329-346.

Paskovic, I., Perica, S., Pecina, M., Hancevic, K., Paskovic, M.P., Custic, M.H. 2013. Leaf mineral concentration of five olive cultivars grown on calcareous soil. Journal of Central European Agriculture, 14.

Pazos, M., Alonso, A., Sánchez, I., Medina, I. 2008. Hydroxytyrosol prevents oxidative deterioration in food stuffs rich in fish lipids. Journal of Agricultural and Food Chemistry, 56: 3334-3340.

Roman, R.A., Amoros, J.A., de los Reyes, C.P., Navarro, F.J.G., Bravo, S. 2014. Major and trace element content of olive leaves. Olivae, 119: 1-7.

Salah, M.B., Abdelmelek, H., Abderraba, M. 2012. Study of phenolic composition and biological activities assessment of olive leaves from different varieties grown in Tunisia. Medical Chemistry, 2: 
107-111.

Salta, F.N., Mylona, A., Chiou, A., Boskou, G., Andrikopoulos, N.K. 2007. Oxidative stability of edible vegetable oils enriched in polyphenols with olive leaf extract. Food Science and Technology International, 13: 413-421.

Sanchez-Moreno, C. 2002. Methods used to evaluate the free radical scavenging activity in foods and biological systems. Food Science and Technology International, 8: 121-137.

Toplu, C., Uygur, V., Yildiz, E. 2009. Leaf mineral composition of olive varieties and their relation to yield and adaptation ability. Journal of Plant Nutrition, 32: 1560-1573.

Yaghoubi, M.J., Gh, G., Satari, R. 2007. Antimicrobial activity of Iranian propolis and its chemical composition. DARU Journal of Pharmaceutical Sciences, 15: 45-48.

Yateem, H., Afaneh, I., Al-Rimawi, F. 2014. Optimum conditions for oleuropein extraction from olive leaves. International Journal Applied Science and Technology, 4: 153-157. 